\title{
BMJ Open Should empiric antibiotic therapy be withheld when aetiology of preterm birth is non-infectious? A protocol for a systematic review
}

\author{
Joanna Seliga-Siwecka, ${ }^{1}$ Mohammed Toseef Ansari, ${ }^{2}$ Judy Aschner, ${ }^{3}$ \\ Margaret Sampson, ${ }^{4}$ Justyna Romańska ${ }^{1}$
}

To cite: Seliga-Siwecka J, Ansari MT, Aschner J, et al. Should empiric antibiotic therapy be withheld when aetiology of preterm birth is non-infectious? A protocol for a systematic review. BMJ Open 2018;8:e018782. doi:10.1136/ bmjopen-2017-018782

- Prepublication history and additional material for this paper are available online. To view these files, please visit the journal online (http://dx.doi. org/10.1136/bmjopen-2017018782).

Received 21 July 2017 Revised 27 February 2018 Accepted 6 March 2018

Check for updates

${ }^{1}$ Department of Neonatology and Neonatal Intensive Care, Medical University of Warsaw, Warsaw, Poland

${ }^{2}$ School of Epidemiology, Public Health and Preventive Medicine, Faculty of Medicine, University of Ottawa, Ottawa, Ontario,

Canada

${ }^{3}$ Department of Pediatrics, Albert Einstein College of Medicine and The Children's Hospital at Montefiore, Bronx, New York, USA

${ }^{4}$ Children's Hospital of Eastern Ontario, Ottawa, Ontario, Canada

Correspondence to

Dr Justyna Romańska;

justyna_romanska@gazeta.pl

\section{ABSTRACT}

Introduction Preterm birth (PTB) at $<37$ weeks of gestation is the leading cause of perinatal morbidity and mortality in developed countries. The traditional approach has been based on the assumption that PTB is primarily a result of intrauterine infection, which triggers preterm labour and puts the newborn at risk of early onset sepsis (EOS). We are currently experiencing a rise in prematurity that results from maternal and fetal diseases unrelated to infection. We have designed a systematic review to assess whether chemoprophylaxis should be withheld when the aetiology of preterm birth is non-infectious.

Methods and analysis Our study will focus on studies evaluating EOS in preterm infants. We will conduct a comprehensive search of literature available up to 28 February 2018. An information specialist will search for eligible studies in Medline (Ovid interface, 1948 and onwards), Embase (Ovid interface, 1980 onwards) and the Cochrane Central Register of Controlled Trials (Wiley interface, current issue). We will search databases and registries including records of ongoing research, conference proceedings and thesis (clinical trials, WHO International Clinical Trials Registry Platform). Two authors will independently extract data from eligible studies and assess risk of bias. For continuous outcomes, which follow discrete distribution, mean difference will be calculated. Dichotomous data will be presented using risk ratios, while count data will be expressed using rate ratios. Time-toevent outcomes will be reported as HRs. All estimates will be presented together with $95 \% \mathrm{Cl}$. Studies comparable with respect to methodology and reporting the same outcomes will be combined in a meta-analysis.

Ethics and dissemination Our systematic review does not require approval from the research and ethics board. We will use the findings to prepare a future multicentre randomised control trial in order to establish safe and adequate antibiotics policies for preterm infants, based on the aetiology of PTB.

PROSPERO registration number CRD42016029707.

\section{INTRODUCTION}

Preterm birth at less than 37 weeks of gestation is the leading cause of perinatal morbidity and mortality in developed countries. Despite
Strengths and limitations of this study

- This is the first systematic review to evaluate the use of empiric antibiotics in preterm babies born for non-infectious reasons.

- Heterogeneity of study settings, design and missing data may influence results.

ongoing improvements in perinatal care, the frequency of preterm delivery remains high (11.4\% in the USA and 5\%-9\% in Europe and other developed countries).

The traditional approach has been based on the assumption that preterm birth is primarily a result of intrauterine infection, which triggers preterm labour and puts the newborn at risk of early-onset sepsis (EOS). Hence, to treat EOS, all preterm infants should receive empiric antibiotics until negative culture results exclude infection. However, we are currently experiencing a rise in the rate of prematurity, which is mainly a result of maternal or fetal conditions unrelated to infection (assisted reproductive technologies and multiple gestation, pre-eclampsia, maternal obesity and diabetes, intrauterine growth restriction (IUGR)). While there are limited data establishing the risk of EOS in this group of preterm infants, there is growing evidence of adverse effects of early exposure to antibiotics on neonatal outcomes; reduced diversity of the newborn microbiome, increased risk of late-onset sepsis (LOS), necrotising enterocolitis (NEC), poor neurological outcomes and death. ${ }^{1-3}$ Additional adverse consequences include impaired maternal-newborn bonding, delayed breast feeding, increased risk of intravenous infiltrates, aminoglycoside toxicity and ototoxicity, and prolonged length of hospital stay that increases healthcare costs. We propose that there is a group of infants who are at low risk of EOS, and for these 
infants, relative risk versus benefit of chemoprophylaxis within the first 48 hours of life is unknown.

Moreover, it is critical to provide physicians with up-todate guidelines regarding implementation of antibiotic therapy in preterm infants, stratified by risk of EOS. No clear guidelines exist for preterm infants $<32$ weeks, ${ }^{45}$ nor are there published systemic reviews on the use of antibiotics in preterm infants born for reasons that are associated with a low likelihood of infection.

\section{OBJECTIVES}

The first aim of this systematic review is to investigate whether infectious versus non-infectious aetiologies of preterm birth lead to different adverse neonatal outcomes. Second, we plan to assess whether there are differences in comparative effectiveness/harms of empiric antibiotic therapy for the two aetiologies.

- Q1. Are there any risk prediction models for EOS, LOS, NEC, length of hospital stay (LOHS), neonatal death or poor neurodevelopmental outcomes developed exclusively for preterm births $\leq 32$ weeks of gestation? If yes, was infectious/non-infectious aetiology of preterm birth evaluated as a predictor in the model(s)? If yes, is infectious/non-infectious aetiology an independent predictor for one or more of these adverse outcomes?

- Q2. When non-infectious indications of preterm birth $\leq 32$ weeks of gestation are compared with infectious indications, what is the relative risk (or odds/ hazards) of EOS, neonatal death, LOS, LOHS, NEC and poor neurodevelopmental outcomes?

- Q3. For births $\leq 32$ weeks' gestation, what is the comparative effectiveness and harm of no, short ( $\leq 72$ hours), medium ( $>72$ hours to $\leq 7$ days) or longer-term ( $>7$ days) empiric antibiotic therapy for infectious and non-infectious aetiologies of preterm birth? Are there important differences in comparative effectiveness between the two aetiologies?

\section{METHODS AND ANALYSES \\ Types of studies}

We will consider primary studies with the following designs:

- Prospective or retrospective cohorts (including cohorts obtained from randomised controlled trials (RCTs)), nested case-control, case-cohort studies or administrated database/registries.

- All types of prediction model studies, that is, model development studies with/without validation, model validation studies, model re-development or updating studies.

Review articles, cross-sectional and case-control designs and models predicting composite outcomes, case reports and case series will be excluded.

\section{Study settings}

Studies conducted worldwide. We plan to conduct separate analyses for developed and developing nations.

\section{Types of interventions}

The study will focus on infectious and non-infectious aetiologies of preterm birth, and evaluate short, medium and long exposure to antibiotics. Infectious aetiology of preterm birth will be defined by maternal symptoms of chorioamnionitis as outlined by the American College of Obstetrics and Gynaecology such as maternal fever $\geq 38^{\circ} \mathrm{C}$ and two of the following: fetal tachycardia $\left(>160 /{ }^{\prime}\right)$, maternal tachycardia $>80 /{ }^{\prime}$, uterine tenderness, maternal leucocytosis $>15 \times 10^{6}$ and foul-smelling discharge. ${ }^{6}$ Histological evidence of chorioamnionitis is present in $>70 \%$ of women who become febrile after an epidural (a common procedure during labour). Despite the lack of other symptoms, these cases will also be considered at risk of EOS together with preterm premature rupture of membranes, preterm labour and maternal colonisation with group B streptococcus. ${ }^{4}$ Non-infectious reasons will include causes such as IUGR, fetal distress, maternal pre-eclampsia (haemolysis, elevated liver enzymes, low platelet count) and placental abruption. Antibiotic exposure will be defined as short ( $\leq 72$ hours), medium ( $>72$ hours to $\leq 7$ days) or longer-term ( $>7$ days) empiric therapy.

\section{Types of outcome measures}

\section{Primary outcomes}

- EOS defined as positive blood or cerebral spinal fluid within the first $48-72$ hours of life. ${ }^{4}$

- LOS defined as positive blood or cerebral spinal fluid after 72 hours of life. ${ }^{7}$

- NEC according to Bell's criteria. ${ }^{8}$

\section{Secondary outcomes}

- Length of hospital stay.

- Neonatal death.

- Poor neurodevelopmental outcomes.

Data extraction items will include funding, geographical region of study, study characteristics (eg, sample size, duration of follow-up and funding); population characteristics and eligibility criteria; intervention characteristics (eg, type of antibiotics, dose, frequency, duration); exposure definitions, measurement tool and cut-offs; number randomised into each group and number analysed; number exposed and unexposed; missing data and reasons for missing data; outcome definition, time point, measurement tool employed, cut-offs and metric; statistical analysis and adjustments; and items necessary to assess risk of bias. For question 1, other data extraction items reported in the CHARMS checklist (CH ecklist for critical A ppraisal and data extraction for systematic $\mathbf{R}$ eviews of prediction $\mathbf{M}$ odelling $\mathbf{S}$ tudies) for risk prediction models will also be extracted. ${ }^{9}$

One reviewer will extract data. Another reviewer will verify outcomes data independently. Discrepancies will be cross-checked against the full text of the record and, where applicable, data entries will be corrected.

A table presenting review eligibility criteria is presented in online supplementary appendix 1 . 


\section{Search methods for identification of studies}

Studies will be identified through searches of bibliographic databases and trial registries, cited and citing references, contacting experts and general Internet searching. Database search strategies will be developed by a librarian experienced in systematic review searching. The MEDLINE strategy will be developed first, with input from the research team. The search will then be adapted for the other databases. Medline, Embase and Cochrane Central Register of Controlled Trials will be searched using the Ovid platform. ClincialTrials.gov and WHO International Clinical Trials Registry Platform will be searched to identify in progress or completed but not yet published trials. Searches will be restricted to the last 30 years, but no study design or language restrictions will be imposed. We will include articles available by 28 February 2018. As preliminary searches suggest that approximately $15 \%$ of the records are published in a language other than English, studies in languages other than English will be excluded during screening as described below. The search will be updated towards the end of the review, after being validated to ensure that the Medline strategy retrieves a high proportion of eligible studies found through any means but indexed in Medline. A draft Medline search strategy is included in online supplementary appendix 2 .

\section{Data collection and analyses}

Selection of studies

Records identified through searching will be imported into Reference Manager where duplicate records will be removed. The remaining records will be uploaded to CrowdscreenSR (InsightScope), a website for crowdsourcing systematic reviews, which enables cooperation between reviewers during the study selection process. The selection process will be piloted by applying the inclusion criteria to a sample of publications to ensure inter-rated reliability. After that, all publications will be assessed independently by two researchers (JSS and JR) at two levels. First, titles and abstracts will be assessed, requiring consensus of two reviewers to exclude a record. Then, full-text articles. Two reviewers assess the full text to determine final eligibility. Disagreements will be resolved by consensus or third-party adjudication.

\section{Data extraction}

An electronic data extraction form will be used to extract key study characteristics (methods, participants and outcomes) (online supplementary appendix 3). Data extraction will be piloted with five randomly selected eligible studies. Data extraction will be performed by two researchers (JSS and JR). Data corrections or amendments will be logged.

\section{Assessment of risk of bias in included studies}

Risk of bias assessment is an assessment of the internal validity of studies. Two reviewers will assess risk of bias independently for each outcome of interest. Disagreements will be resolved by consensus or third-party adjudication.
For question 1, we will use the CHARMS checklist to assess study validity. ${ }^{9}$ For question 2, the Quality In Prognosis Studies tool that covers six domains, namely, study participation, study attrition, prognostic factor measurement, outcome measurement, study confounding and statistical analysis and reporting, will be used. ${ }^{10}$ When evidence for question 3 originates in RCTs, the Cochrane risk of bias tool will be employed. Assessment of risk of bias of non-randomised studies addressing question 3 will be based on our generic assessment of selection bias (including attrition bias or missing data), confounding (including timevarying changes in treatment/confounders), information bias and bias due to use of co-interventions. ${ }^{11}$ Risk of bias assessment will be undertaken for all biassing domains, and each will be judged as high, moderate, low or unclear risk of bias. Overall risk of bias of a study will be judged across domains as high, moderate or low. A domain rating of high risk of bias will automatically lead to a judgement of high overall risk of bias. When no domain-specific assessment of the risk of bias was rated as high, the study's overall risk of bias will not be judged as high.

Publication bias will be investigated for the body of evidence from randomised controlled trials if the following criteria are met $^{12}$ :

- $\geq 10$ studies contributing data for an outcome.

- Studies of unequal sizes.

- No substantial clinical and methodological differences between smaller and larger studies.

- Quantitative results accompanied by measures of dispersion.

\section{Applicability}

Characterisation of study applicability or generalisability will be made by two reviewers and categorised as major concerns, minor concerns or no concern with corresponding rationales documented. Determinants of applicability incorporate population characteristics, study environmental settings, intervention dose/frequency/ timing, definition of outcomes and exposures and their measurement techniques, adequacy of follow-up and background standards of care.

\section{Measures of treatment effect}

Question 1 investigates whether infectious and non-infectious aetiologies of birth $\leq 32$ weeks would remain a significant predictor of a number of adverse neonatal and longer-term health outcomes in risk prediction models including other candidate predictors. This will be answered through a descriptive synthesis of relevant literature, informed by judgements of overall study risk of bias. No quantitative data pooling of these statistical estimates will be conducted. We will report the parameters of models from studies assessed as low risk of bias and generalisable models.

For question 2, the decision to conduct a meta-analysis will be based on an assessment of between-study heterogeneity, measured by the $\mathrm{I}^{2}$ statistic. Between-study heterogeneity (ie, $\mathrm{I}^{2}>50 \%$ ) can be explained by study-level 
clinical or methodological covariates. Statistical heterogeneity between studies will be quantified with $\mathrm{I}^{2}$ statistics and the $\mathrm{P}$ value from the $\chi^{2}$ test $(\mathrm{P} \leq 0.10)$ will be used to determine statistical significance. As well as statistically heterogeneity, clinical and methodological heterogeneity will be explored with methodological covariates including study design, study risk of bias and funding and clinical covariates such as the severity of preterm birth, Apgar scores, antibiotic therapy protocol, mode of delivery and specific laboratory testing protocols. Such heterogeneity would be investigated in subgroup analyses or meta-regression. Where meta-analysis is warranted, data will be pooled using random-effects generic inverse variance or Mantel-Haenszel method because group sizes are likely to be different in contributing observational studies. Other random-effects models (eg, Peto odds or inverse variance method) may be used as recommended by previously published guidance. ${ }^{13}$ Adjusted estimates of association will be preferentially selected over crude estimates in meta-analyses. Pooled data will be reported as OR, HR, relative risk or mean difference. Sensitivity analyses by study risk of bias will be undertaken as required. Evidence originating in studies that used prophylactic/ empiric maternal or neonatal antibiotic therapy will be synthesised separately from those that did not. Post hoc subgroup analyses may be undertaken if warranted by the data.

Approach to meta-analysis for evidence pertaining to question 3 will be similar to the approach described for question 2. RCT data will not be combined with non-randomised studies. Sparse data will not be included in the meta-analysis but rather described narratively. Studies with zero events in both arms will be excluded from the meta-analysis.

\section{Data synthesis}

For continuous outcomes, which follow discrete distribution, mean difference will be calculated. Dichotomous data will be presented using relative risk, while count data will be expressed using rate ratios. Time-to-event outcomes will be reported as HRs. All estimates will be presented together with $95 \%$ CIs.

Studies comparable with respect to methodology and reporting the same outcomes will be combined in a meta-analysis. Between-study heterogeneity will be examined using the $\chi^{2}$ test and the $\mathrm{I}^{2}$ statistics. Fixed-effect meta-analysis will be permitted only when $P$ value of $\chi^{2}$ test $>0.1$ and $\mathrm{I}^{2}<40 \%$ indicating that the betweenstudies differences are not statistically significant and observed heterogeneity might not be important. ${ }^{11} 14$ Random-effect meta-analysis will be carried out using either DerSimonian and Laird or inverse variance methods of weight assignment for either continuous data or all remaining outcomes. ${ }^{14}$ Fixed-effect meta-analysis will be conducted using the algorithm proposed by Mantel and Haenszel as well as the inverse variance method for cardinal and all other types of outcomes, respectively. ${ }^{15}$ Significance of the overall effect will be tested with two-tailed Z-test assuming $\mathrm{P}<0.05$ as the level of significance.

Qualitative synthesis with either narrative description or tabular representation will be presented when studies could not be quantitatively combined due to unacceptable heterogeneity of missing data precluding meta-analysis.

All statistical analyses will be conducted using dedicated software. R statistical software with 'metafor' package will preferentially be used for all calculations and generation of corresponding plots, but the use of other statistical programs cannot be excluded. ${ }^{16}$

\section{Assessment of certainty of evidence}

For each outcome, when prognostic or effect estimates are not very wide and can be conclusively interpreted, we will grade the certainty of evidence as per the published GRADE approach (Grading of Recommendations, Assessment, Development, and Evaluation-GRADE). ${ }^{17} 18$ For example, if for question 3, short duration of empiric antibiotic therapy versus long-term therapy yields wide CI for the outcome NEC, such that one cannot exclude the possibility that short duration may be harmful, equivalent or superior to long term, then results are inconclusive and, as such, grading of the certainty of evidence will not be attempted for this outcome.

\section{Dealing with missing data}

The data will be analysed on an intention-to-treat principle. In case of missing data, which preclude inclusion of the outcome into quantitative accumulation, we will attempt to contact the corresponding author in order to obtain required information. The extent and implications of missing data will be reported.

\section{Assessment of heterogeneity and sensitivity analysis}

Between-study heterogeneity will be examined using the $\chi^{2}$ test and the $\mathrm{I}^{2}$ statistics, as described above. When between-trials variability reaches statistical significance ( $\mathrm{P}$ value for heterogeneity $<0.1$ ), attempts to explain heterogeneity will be undertaken using sensitivity analyses with either subgroup meta-analysis or meta-regression. Factors or continuous measures that may potentially influence the results will be analysed as covariates. For subgroup meta-analysis, identified studies will be stratified according to the following explanatory variables: study quality, race (black vs non-black), ethnicity (Hispanic vs non-Hispanic) or region (developing vs developed). Between-subgroup effects will be assessed using the test for interaction as proposed by Borenstein $e t$ $a l$ with $\mathrm{P}<0.05$ indicating statistically significant impact of the covariate on observed effect size.$^{19}$ The contribution of continuous covariates (ie, mean maternal age, mean gestational age at delivery, per cent of Black infants, mean birth weight, mean Apgar score) to between-study heterogeneity will be explored with random-effect meta-regression provided that at least 10 studies will be available for each explanatory variable. ${ }^{11}$ 


\section{Assessment of publication biases}

For meta-analyses with at least 10 studies, the risk of publication bias will be examined by visual inspection of funnel plots and statistically assessed with the use of both Egger's and Begg's tests with $\mathrm{P}<0.05$ considered statistically significant.

\section{Patient and public involvement}

Patients and public were not involved in the study.

\section{DISCUSSION}

By conducting this systematic review, we plan to establish whether preterm infants born at $\leq 32$ weeks of gestation due to non-infectious reasons should receive prophylactic antibiotic therapy. We will use the findings of this systematic review to prepare a future multicentre randomised control study in order to establish safe and adequate antibiotics policies for extreme, severe or moderately preterm infants. Furthermore, we will provide up-to-date evidence of the harms and benefits of chemoprophylaxis in the most premature group of newborns. Additionally, we plan to discuss how our findings may be applied in future guidelines and hospital policies.

\section{ETHICS AND DISSEMINATION}

We did not submit for ethical approval, as the study does not include individuals. All significant modifications in the protocol will be reported to PROSPERO. The full protocol will be widely available due to open access. We plan to submit our findings to international peer-reviewed journals (paediatric, infectious, epidemiology). Abstracts will be submitted to local and international conferences.

The systematic review will be used to prepare a multicentre prospective trial with the aim of evaluating the safety of using a more targeted antibiotic approach in low-risk preterm infants, including a delay in antibiotic initiation until laboratory tests results and blood culture results are available.

Acknowledgements We would like to thank Dr Dayre McNally from the Department of Pediatrics, Children's Hospital of Eastern Ontario, and Nassr Nama from the Faculty of Medicine, University of Ottawa, for providing us with CrowdScreenSR (InsightScope).

Contributors JS-S, MTA and JR conceptualised and designed the study, drafted the initial manuscript and approved the final manuscript as submitted. JA critically reviewed the manuscript and approved the final manuscript as submitted. MS developed the electronic search strategies, reviewed and revised the manuscript, and approved the final manuscript as submitted.

Funding This research received no specific grant from any funding agency in the public, commercial or not-for-profit sectors.
Competing interests None declared.

Patient consent Not required.

Provenance and peer review Not commissioned; externally peer reviewed.

Open Access This is an Open Access article distributed in accordance with the Creative Commons Attribution Non Commercial (CC BY-NC 4.0) license, which permits others to distribute, remix, adapt, build upon this work non-commercially, and license their derivative works on different terms, provided the original work is properly cited and the use is non-commercial. See: http://creativecommons.org/ licenses/by-nc/4.0/

(C) Article author(s) (or their employer(s) unless otherwise stated in the text of the article) 2018. All rights reserved. No commercial use is permitted unless otherwise expressly granted.

\section{REFERENCES}

1. Cotten CM, Taylor S, Stoll B, et al. Prolonged duration of initial empirical antibiotic treatment is associated with increased rates of necrotizing enterocolitis and death for extremely low birth weight infants. Pediatrics 2009;123:58-66.

2. Kuppala VS, Meinzen-Derr J, Morrow AL, et al. Prolonged initial empirical antibiotic treatment is associated with adverse outcomes in premature infants. J Pediatr 2011;159:720-5.

3. Alexander VN, Northrup V, Bizzarro MJ. Antibiotic exposure in the newborn intensive care unit and the risk of necrotizing enterocolitis. $J$ Pediatr 2011;159:392-7.

4. Polin RA. Committee on Fetus and Newborn. Management of neonates with suspected or proven early-onset bacterial sepsis. Pediatrics 2012;129:1006-15.

5. Tripathi N, Cotten CM, Smith PB. Antibiotic use and misuse in the neonatal intensive care unit. Clin Perinatol 2012;39:61-8.

6. Tita AT, Andrews WW. Diagnosis and management of clinical chorioamnionitis. Clin Perinatol 2010;37:339-54.

7. Tsai MH, Hsu JF, Chu SM, et al. Incidence, clinical characteristics and risk factors for adverse outcome in neonates with late-onset sepsis. Pediatr Infect Dis J 2014;33:e7-e13.

8. Bell RS, Graham CB, Stevenson JK. Roentgenologic and clinical manifestations of neonatal necrotizing enterocolitis. Experience with 43 cases. Am J Roentgenol Radium Ther Nucl Med 1971;112:123-34.

9. Moons KG, de Groot JA, Bouwmeester W, et al. Critical appraisal and data extraction for systematic reviews of prediction modelling studies: the CHARMS checklist. PLoS Med 2014;11:e1001744.

10. Hayden JA, van der Windt DA, Cartwright JL, et al. Assessing bias in studies of prognostic factors. Ann Intern Med 2013;158:280-6.

11. GSe H. Cochrane handbook for systematic reviews of interventions version 5.1.0. Oxford, UK: The Cochrane Collaboration, 2011.

12. Rühmann I, Maier C. [The modification of injection pain and the incidence of thrombophlebitis following etomidate]. Anasth Intensivther Notfallmed 1990;25 Suppl 1(Suppl 1):31-3.

13. Fu R, Gartlehner G, Grant M, et al. Conducting quantitative synthesis when comparing medical interventions: AHRQ and the effective health care program. J Clin Epidemiol 2011;64:1187-97.

14. DerSimonian R, Laird N. Meta-analysis in clinical trials. Control Clin Trials 1986;7:177-88.

15. Mantel N, Haenszel W. Statistical aspects of the analysis of data from retrospective studies of disease. J Natl Cancer Inst 1959;22:719-48.

16. Viechtbauer W. Conducting meta-analyses in $R$ with the metafor package. J Stat Softw 2010;36:1-48.

17. Balshem $\mathrm{H}$, Helfand $\mathrm{M}$, Schünemann $\mathrm{HJ}$, et al. GRADE guidelines: 3 . Rating the quality of evidence. J Clin Epidemiol 2011;64:401-6.

18. Iorio A, Spencer FA, Falavigna M, et al. Use of GRADE for assessment of evidence about prognosis: rating confidence in estimates of event rates in broad categories of patients. BMJ 2015;350:h870.

19. Borenstein MHL, Higgins J, Rothstein H. Introduction to metaanalysis. Chichester, UK, 2009. 